\title{
Analysis of mutations in the tudor domain of the survival motor neuron protein SMN
}

\author{
Payam M ohaghegh ${ }^{1}$, Nanda R R odrigues ${ }^{1}$, Nicholas $\mathrm{O}_{\text {wen }}{ }^{1}$, Christopher P Ponting ${ }^{3}$, \\ Thanh T L $e^{2}$, A rthur H M Burghes ${ }^{2}$ and $K$ ay E Davies ${ }^{1}$ \\ ${ }^{1} \mathrm{D}$ epartment of $\mathrm{H}$ uman A natomy and G enetics, U niversity of Oxford, UK \\ ${ }^{2} \mathrm{D}$ epartment of N eurology, O hio State University, Columbus, USA \\ ${ }^{3}$ Current address: $N$ ational Center for B iotechnology Information, $N$ ational Institutes of $H$ ealth, B ethesda, M D \\ 20894, USA
}

\begin{abstract}
A utosomal recessive childhood onset spinal muscular atrophy (SMA) is a leading cause of infant mortality caused by mutations in the survival motor neuron (SMN) gene. The SMN protein is involved in RNA processing and is localised in structures called GEMs in the nucleus. Nothing is yet understood about why mutations in SM N gene result in the selective motor neuron loss observed in patients. The SM N protein domains conserved across several species may indicate functionally significant regions. E xon 3 of SM N contains homology to a tudor domain, where a Type I SM A patient has been reported to harbour a missense mutation. We have generated missense mutants in this region of SM N and have tested their ability to form GE M s when transfected into $\mathrm{HeL}$ a cells. $\mathrm{O}$ ur results show such mutant SM N proteins still localise to G E M s. Furthermore, exon 7 deleted SM N protein appears to exert a dominant negative effect on localisation of endogenous SM N protein. H owever, exon 3 mutant protein and exon 5 deleted protein exert no such effect.
\end{abstract}

Keywords: spinal muscular atrophy; survival motor neuron; gemini of coiled bodies; tudor domains

\section{Introduction}

Childhood onset spinal muscular atrophy (SMA) is a leading cause of infant mortality, which segregates as an autosomal recessive disease with a carrier frequency of 1 in $60 .{ }^{1}$ The disorder is characterised by the loss of lower motor neurones in the spinal cord and proximal muscle wasting. The disease is categorised into three types according to the severity and age of onset: Type I (Werdnig-H offmann disease) has an onset either in

Correspondence: Professor Kay E Davies, Department of $\mathrm{H}$ uman A natomy and G enetics, U niversity of Oxford, South Parks R oad, O xford, OX $13 Q$ X. U K. Tel: (0)1865 272416; Fax: (0) 1865 272427; E-mail: kay.davies@anat.ox.ac.uk

R eceived 28 January 1999; revised $17 \mathrm{M}$ arch 1999; accepted $19 \mathrm{M}$ arch 1999 utero or shortly after birth and death usually occurs before the age of 2 years; Typell SMA has an onset after 2 years of age where individuals are never able to stand but may sit unaided; TypellI (KugelbergWelander) SM A patients are able to stand unaided, and onset is often in adolescence. ${ }^{2} A$ II three forms of SM A are localised to chromosome $5 q 11.2-q 13.3$ in a region containing an inverted duplication and at least three genes (for review see L efebvre ${ }^{3}$ ). A Ithough deletions encompassing all these genes are found in SMA patients, ${ }^{4-6}$ it is now well established that survival motor neuron (SMN) gene is the causative gene. ${ }^{3}$ SMN is present in two almost identical copies in the region: the more telomeric copy of the gene is designated SM N 1 and the centromeric one SMN2. The SMN1 gene is mutated or gene converted to the SM N2 sequence in 
90-98\% of patients (for review see Burghes ${ }^{7}$ ). The SM N 2 gene is deleted in 3-5\% of normal individuals and is therefore not associated with the disease., ${ }^{4,6}$

The SM N gene contains domains and motifs indicative of a role in RNA processing. For example, it contains a $Y G$ box motif which when mutated causes SMA. ${ }^{8}$ This $Y G$ box is well conserved in S.pombe, C. elegans and mammalian SMN orthologues, and has been shown to be important for the oligomerisation of SM N. ${ }^{9}$ Studies by D reyfuss and colleagues ${ }^{10-13}$ have demonstrated directly that SM N plays a role in R NA processing. The SM N protein localises to the cytoplasm and the nucleus in $\mathrm{HeL}$ a cells and is associated with RNA processing proteins by yeast two-hybrid analysis using the RGG box of the hnRNP $U$ protein as bait. ${ }^{10}$ Subsequently, antibodies raised against SM N 1 protein showed that SM N 1 in the nucleus is found in structures called GEMs (Gemini of Coiled Bodies) which are associated with coiled bodies, believed to be involved in mRNA metabolism. ${ }^{10,11,12}$ The dominant negative mutant SMN (SM N delN 27) causes reorganisation of snR NPs in the nucleus as well as inhibiting pre-mR NA splicing in vitro. High levels of full length SMN stimulate splicing. ${ }^{13}$

The number of GEM s seen in the nucleus correlates with the level of full-length protein. These data are consistent with the lower levels of protein seen in Type I patients relative to Type II and Type III patients. ${ }^{14,15}$ SM N has also been shown to be associated with $\mathrm{BCl} 2$ although these data remain to be confirmed by other groups. $^{16}$

Sequence analysis has led to the identification of SM N orthologues in several organisms including $\mathrm{C}$. elegans and S. pombe. ${ }^{8}$ The domains conserved across these species are likely to indicate functionally significant regions. In particular exon 3 , which is proposed to encompass a tudor domain, is highly conserved. ${ }^{17}$ Tudor domains are present in proteins, which have an RNA binding function, suggesting a role in RNA metabolism. ${ }^{18}$

No studies have yet been reported on the effect of mutations in the tudor domain for SM N 1. We therefore decided to express SMN 1 with a missense mutation in exon 3 in $\mathrm{H}$ eL a cells to study its ability to form GEM S. This mutation has been reported in a Typel SMA patient and results in a change of a glutamic acid residue to a lysine. ${ }^{3}$ In addition, we also expressed SMN 1 with substitution of glutamic acid 104, a conserved residue in $S M N 1$, and in a recently described SMN-related protein/SPF 30 found in spliceosomes. ${ }^{18}$
Our data show that substitution of either of these residues does not impair GEM formation.

\section{Experimental Methods}

\section{Mutagenesis}

QuikChange $^{T M}$ Site-Directed M utagenesis (Strategene, A msterdam, Netherlands) system was used to generate E 104A and E 134K using SM N in pCD NA 3 expression vector as template.

HeLa Cells in Culture and Immunofluorescence $\mathrm{HeL}$ a cells were grown and transfected as previously. ${ }^{18}$ Haemagglutinin ( $\mathrm{HA}$ ) antibody (Boehringer $M$ annheim, Mannheim, Germany) was used at 1:1500 dilution. We generated and purified a rabbit polyclonal antibody raised against a peptide corresponding to amino acid residues 170-187 in exon 4 of SM N, (SM N-ex4). SM N-ex4 was used at 1:500 dilution. SMN-C 3 antibody ${ }^{14}$ was used at 1:1000 dilution. A ntibodies were incubated as previously ${ }^{18}$ with $\mathrm{HeL}$ a cells and visualised using fluorescently labelled secondary antibody. Indocarbocyanide (Cy3) and fluorescein isothiocyanate (FITC) were at 1:1000 dilution. 4,6-diamidino2-phenylindole (DA PI) counter staining was carried out by applying DA PI-Vectashield mounting medium and placing in humidity chamber overnight at $4^{\circ} \mathrm{C}$.

\section{Results and Discussion}

In order to study the effect of the patient mutation at position 134 on the SMN1 protein (see Figure 1), a SM N 1 mammalian expression construct was generated. The mutation was introduced using a PCR -based sitedirected mutagenesis approach (see Experimental Methods). A second SMN1 expression construct was generated with a missense mutation at SM N 1 amino acid position 104. This residue is absolutely conserved in human, mouse, zebrafish, S. pombe SM N (Figure 1a), and in human and D rosophila melanogaster SM Nrp (Figure $1 \mathrm{~b}$ ). This construct contained a substitution of a glutamic acid residue 104 to an alanine (E 104A ). These two constructs were used for transfecting $\mathrm{H}$ el a cells to study their localisation in GEMs. The detection of expressed protein in cells was performed using anti-H A antibodies. The controls used were SMN full-length (exon 1-7) construct, SMN exon 7 deleted construct (leading to loss of GEM formation), an SM N exon 5 deleted construct, where GEM formation is not lost, (exon deletion control).

Figure 2 shows the results of the transfection of SM N constructs into $\mathrm{HeL}$ a cells. The missense mutations in exon 3 of SMN clearly do not lead to loss of GEM formation (Figure 2a, 2c). The over-expression of SM N 
A

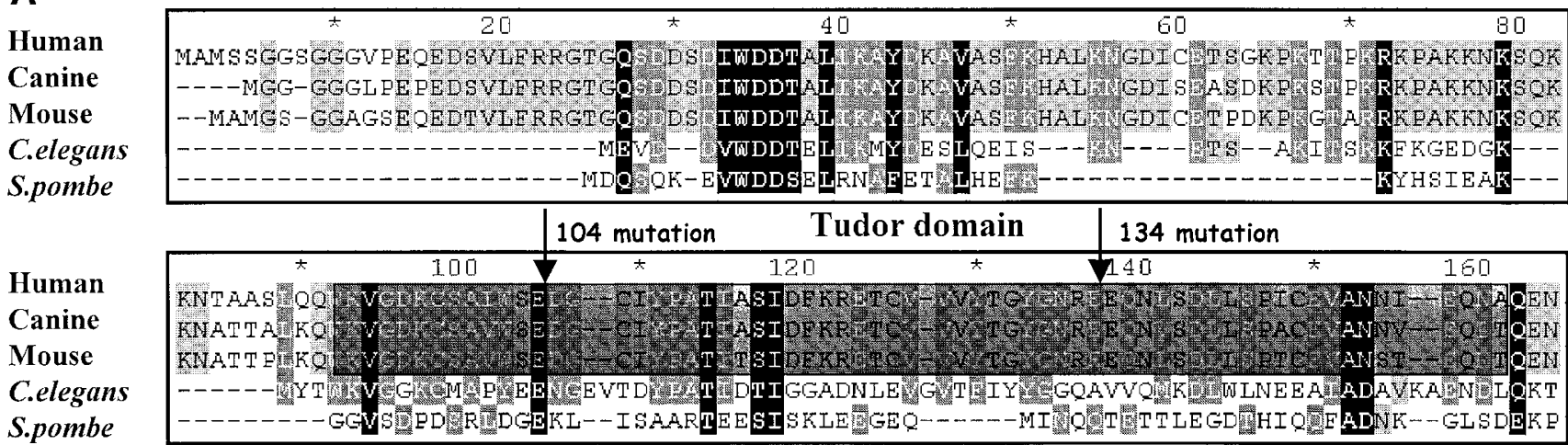

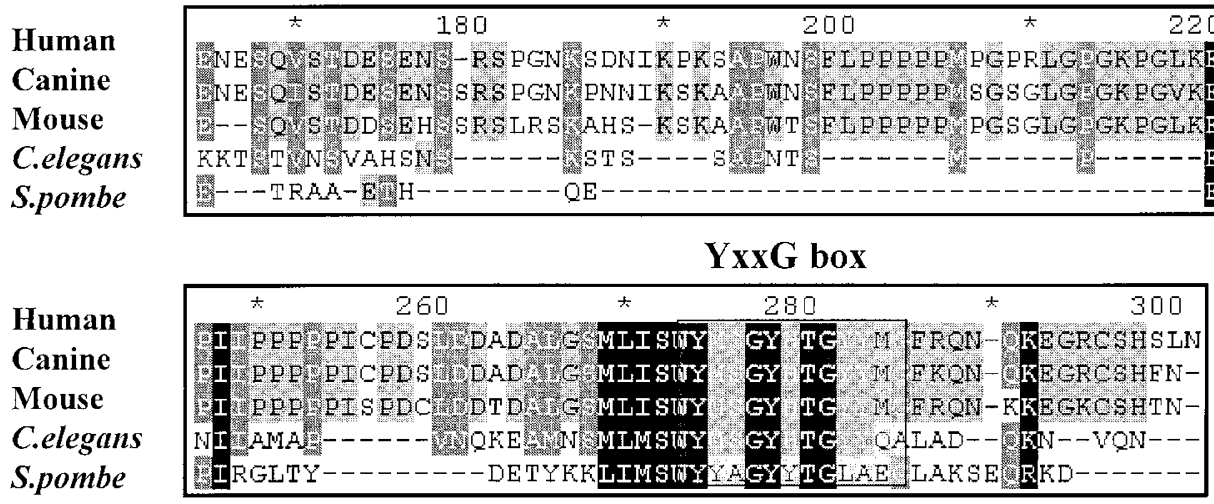

\section{B}

SMNRP-HUMAN $/ 1-303$ SMNRP - DROM $/ / 1-303$ SMN - HUMAN $/ / 1-303$ SMN - HUMAN $/ / 1-303$

SHN RP - HUHAN $/ 1-303$ SMNRP - DROM $/ / 1-303$ SHN - HUHAN $/ 1-303$

\section{SMNRP - HUMAN $/ / 1-303$ SHN RP-DROM $/ / 1-303$} SMN-HUHAN $1 / 1-303$ SMNRP-DROM $/ / 1-303$ SMN - HUMAN $/ 1-303$

SHNRP - HUMAN $/ 1-303$ SHNRP - DROM//1-303 SMN-HUKAN
SMNRP-HUMAN $/>1-303$ SMNRE $-\mathrm{DROH} / / 1-303$

SHNRP - HUMAN $/ / 1-303$

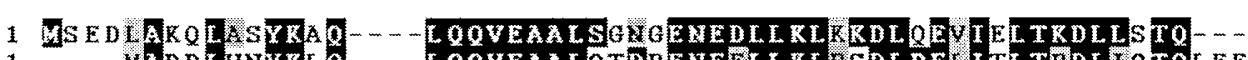

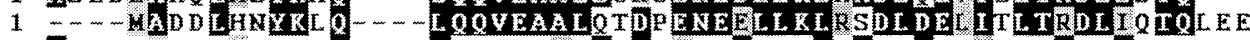

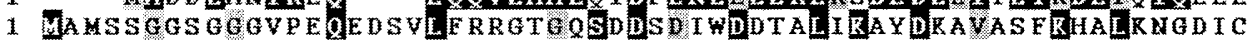
104 mutation

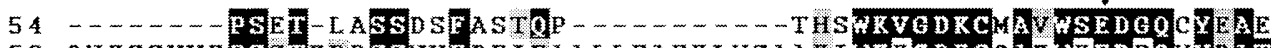

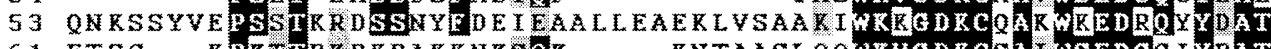

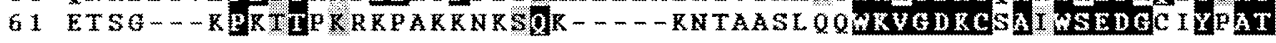

94 TEEIDE NGTAAITFAGYGNAE- - - - VTPLLNLRPVEEGRKAREDSGNRPMSRKEMI 113 I EDTSS-TGEVUVT FDAYQWRS- - - - -TTHVNELR - - -ERT TRUEVE - -DSNKRHRP 113 IASIDFRRETCVVVYTGYGNREEQNL SDLTSPTCEVAN NTEONAOENENESOVSTDESE

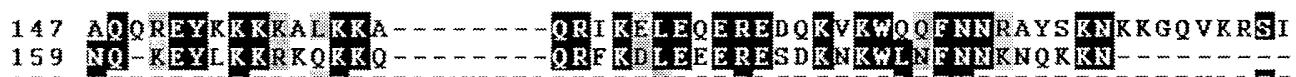

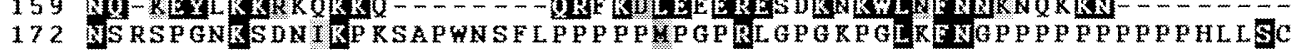

199 FA - - SPESVTGKVGVGTCGIADK PNTQYQDTSRYRVRHL PQ

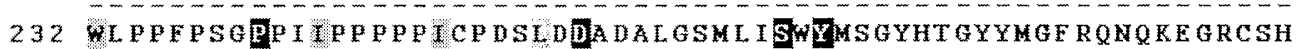

Figure 1 a: M ultiple alignment of SM N orthologous. b: M ultiple alignment of human SM N-related protein (SM NRP), D rosophila melanogaster SM NRP, and human SM N; constructed using Clustal-X; showing the location of missense mutation in the SM N tudor domain, used in over-expression studies. 

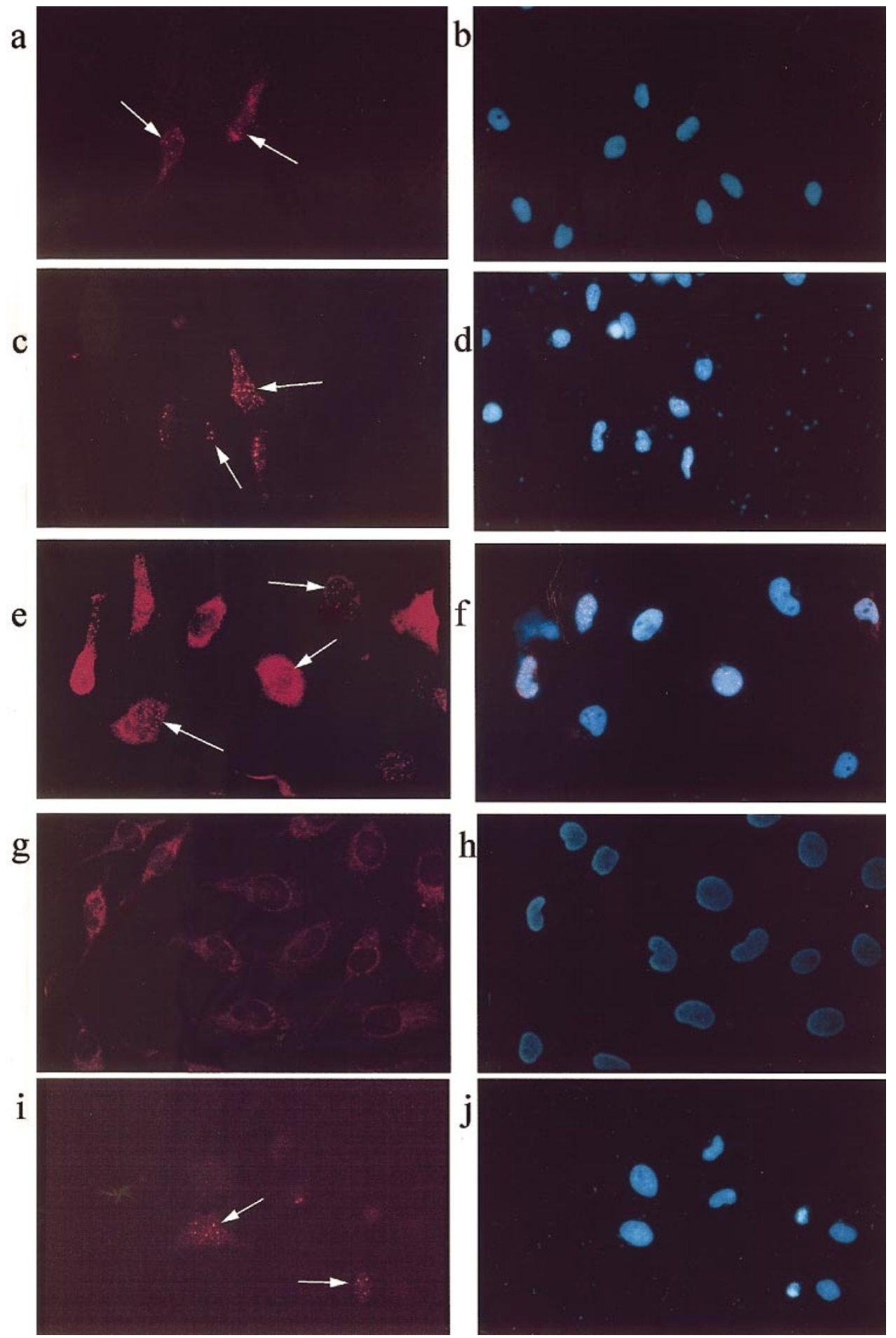

Figure 2 Transfection of H A-tagged-SM N constructs into $\mathrm{H}$ eL a cells (arrows indicate the G E M s). M ag. $400 \times$. a missense mutation E 134K; c missense mutation E 104A; e full length SM N; $\mathbf{g}$ SM N exon 7 deleted; i SM N exon 5 deleted; $\mathbf{b}, \mathbf{d}, \mathbf{f}, \mathbf{h}, \mathbf{j}$ Triple band filter: DA PI (blue) stain showing the nucleus of both transfected and non-transfected cells. The transfected cells over-expressing $\mathrm{H} A$-taggedSM N protein are detected by HA antibody and visualised with secondary Cy3 (red) antibody. 
a

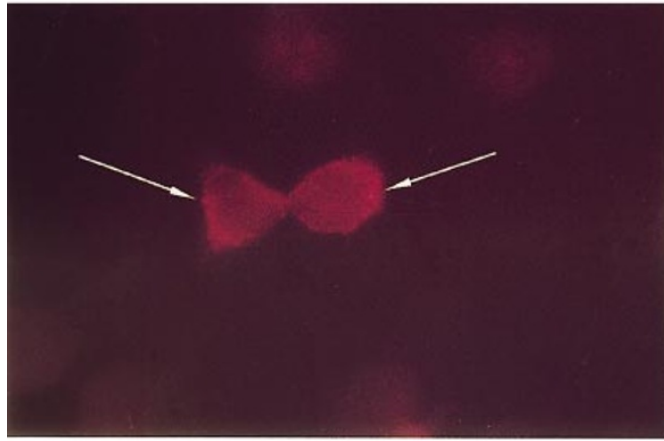

C

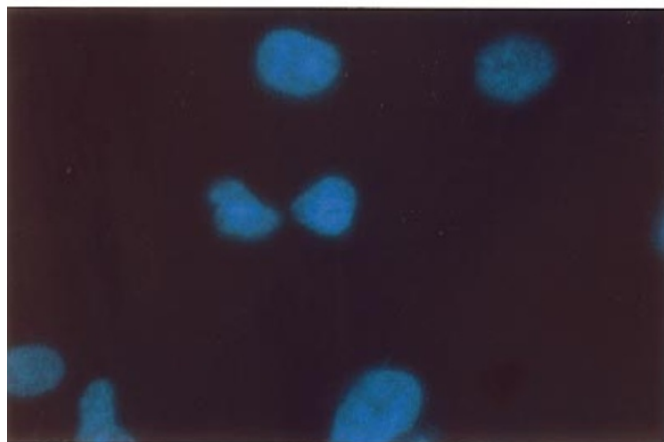

e

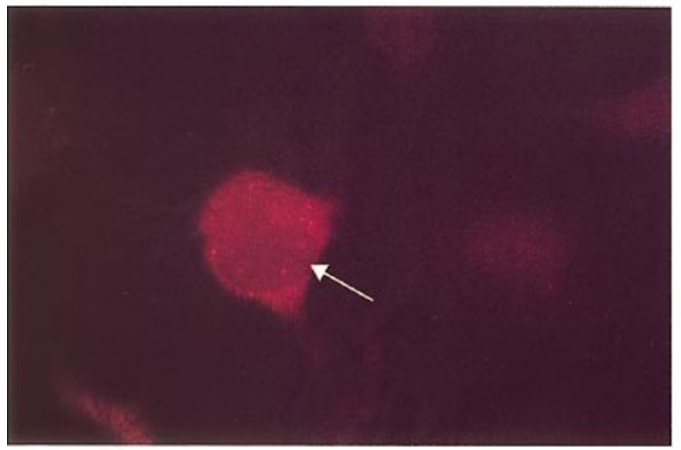

g

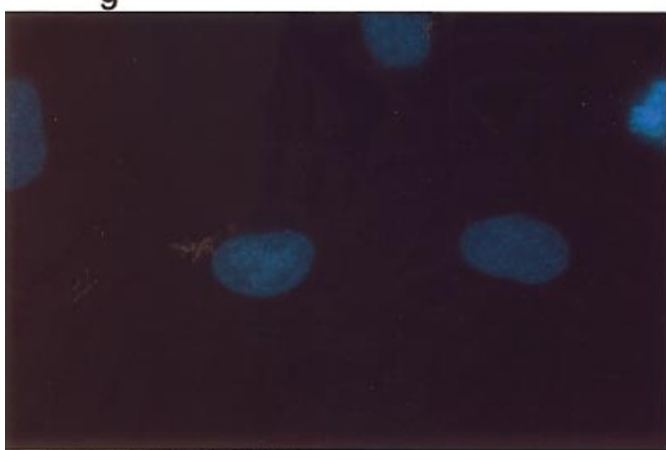

b

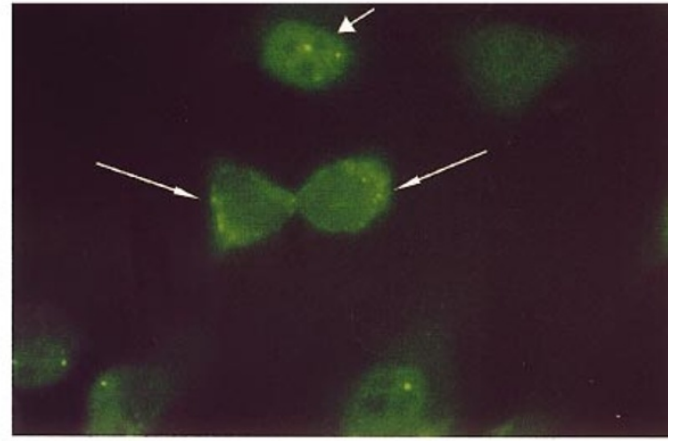

d

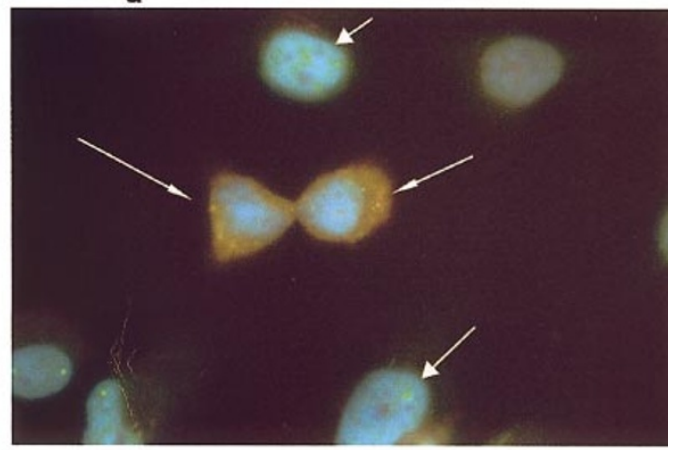

f

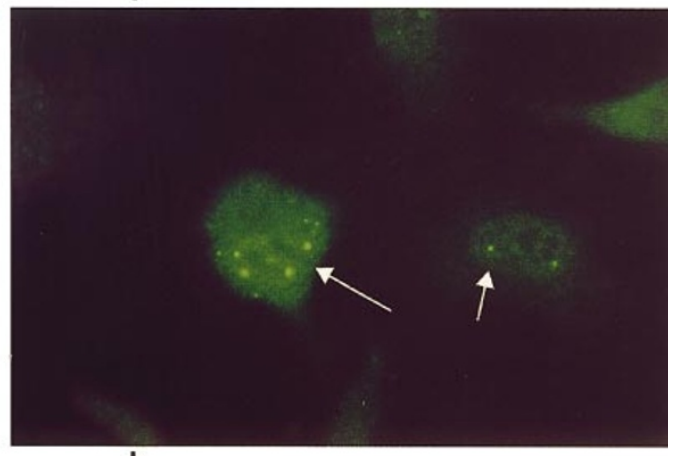

h

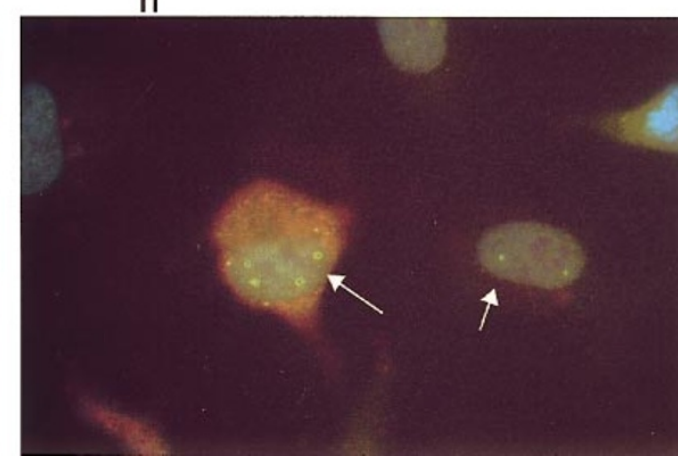

Figure 3 Transfection of $\mathrm{HA}$-tagged-SM N constructs into $\mathrm{H}$ eL a cells. E xamining the effect of over-expression of SM N protein on the endogenous SM N protein. M ag. $1000 \times$. a-d SM N exon 7 deleted construct. The two central cells are over-expressing the SM N 7de leading to loss of G E M formation. The non-transfected cells: top centre and on bottom of picture show endogenous SM N and G E M S detected by SM N-ex4 antibody. e- $\mathbf{h}$ SMN exon 5 deleted construct. Double antibody labelling: a, e showing the over-expressed H A -tagged SM N protein detected by H A antibody and visualised with secondary Cy3 (red) antibody. b, f SM N-ex4 antibody detecting both the endogenous and over-expressed protein, (FITC, green). c, g DAPI stain showing the nucleus of both transfected and nontransfected cells. d, $\mathbf{h}$ Triple band filter. Thick arrows pointing at GE M s, thin arrow pointing at cytoplasmic aggregates. 
proteins also leads to formation of cytoplasmic aggregates. However, deletion of exon 7 abolishes GEM formation consistent with the importance of the $Y G$ bo $\mathrm{x}^{8}$ and residues in this region for SM N oligomerisation. ${ }^{9}$ Furthermore on the same time course, the relative intensity of signal from exon 7 deleted overexpressed SM N protein in the cytoplasm (Figure 2g), to other over-expressed SM N proteins here (Figure 2a, b, $C, 1)$ appears weaker. This might be a reflection on the instability of exon 7 truncated protein or perhaps due to the inability of the protein to be transported across the nuclear member efficiently to form GEMs.

It has been proposed that SMN mutant proteins might exert a dominant negative effect on SMN function. ${ }^{13,16}$ The deletion of the first 27 amino acids of SMN has such an effect. ${ }^{13}$ In SMA patients the ratio between levels of truncated mRNA and protein from SMN2 to full length SMN mRNA ${ }^{4}$ and protein ${ }^{15}$ is increased. The truncated or mutant SM N protein may be able to interact with normal SMN protein and interfere with its function. We studied the overexpression of exon 3 mutant and the exon 5 deleted and exon 7 deleted SMN proteins to determine if they exerted such an effect in vivo. Double antibody labelling of transfected $\mathrm{H}$ eL a cells was carried out.

Figure 3 shows loss of endogenous SM N's ability to form GEMs in the nucleus after over-expression of exon 7 deleted SMN protein. The over-expressed exon 7-deleted SM N has a dominant negative effect on the endogenous SMN, resulting in loss/decrease in number of GEMS. The over-expression of full length SM N 1, E 134K mutant and SM N exon 5 deleted has no such effect and can lead to an increased number of GEMS. The over-expressed SM N tagged proteins were detected with anti-HA antibody (Figure 3 panels (a) and (e)). The SMN-ex4 antibody detected both the over-expressed and the endogenous protein (Figure 3 panels (b), (f)). This allowed tracking and localisation of the over-expressed mutant proteins in relation to normal endogenous protein. There was no detectable difference between the localisation of the overexpressed SM N proteins detected by HA -tagged antibody to endogenous SMN detected by SMN-ex4 antibody. However, over-expression of exon 7 deleted protein seems to interfere with endgenous SM N, as the SM N-ex4 antibody (Figure 3b) detected no GE M s. This appears to be dose dependent as transfected cells weakly staining with $\mathrm{HA}$-tagged antibody still show some GEM formation. H owever the cells with highest expression of SM N7del are unable to form any GEM S.
This provides direct evidence for deleted exon 7 protein exerting a dominant negative effect. The over-expression of E 134K, exon 5 deleted, and full length protein have no such effect, they in fact result in an increased number of G E M s. In addition to SM N-ex4 antibody we also repeated these experiments using $\mathrm{SMN}-\mathrm{C} 3$ antibody, for detection of endogenous SMN protein, and obtained a similar staining pattern.

In conclusion, an exon 3 missense mutation does not inhibit GEM formation, and is unable to exert a dominant negative effect on the formation of these structures. It is likely that the SMN tudor domain performs roles other than in GEM formation. Impairment of one of these functions still results in SMA as seen in one Typel patient. One possibility is that the ability of SMN to bind with one of its interacting proteins is lost. Further studies are under way to determine if mutations in the tudor domain would lead to loss of interaction with any of the SMN interacting proteins.

\section{Acknowledgements}

We are grateful to the M uscular Dystrophy G roup of G reat Britain and Northern Ireland, the Muscular Dystrophy A ssociation USA and the Wellcome Trust for financial support.

\section{References}

1 Pearn J: Incidence, prevalence, and gene frequency studies of chronic childhood spinal muscular atrophy. J Med Genet 1978; 15: 409-413.

2 M unsat TL: Workshop report: international SMA collaboration. Neuromusc D isord 1991; 1: 81.

3 L efebvre S, B urglen L, Frezal J, M unnich A, M elki J : The role of the SM N gene in proximal spinal muscular atrophy. H um Mol G enet 1998; 7: 1531-1536.

4 L efebvre $S$, Burglen L, R eboullet $S$ et al: Identification and characterization of a spinal muscular atrophy-determining gene. Cell 1995; 80: 155-165.

5 R oy N, Mahadevan MS, MCL ean M et al: The gene for neuronal apoptosis inhibitory protein is partially deleted in individuals with spinal muscular atrophy. Cell 1995; 80 167-178.

6 R odrigues NR, O wen N, Talbot K, I gnatius J, D ubowitz V, $D$ avies KE : D eletions in the survival motor neuron gene on $5 q 13$ in autosomal recessive spinal muscular atrophy. $\mathrm{H}$ um M ol G enet 1995; 4: 631-634.

7 Burghes A H M : When is a deletion not a deletion? When it is converted. A m J H um G enet 1997; 61: 9-15.

8 Talbot K, Ponting CP, Theodosiou A M et al: Missense mutation clustering in the survival motor neuron gene: a role for a conserved tyrosine and glycine rich region of the protein in RNA metabolism? Hum Mol Genet 1997; 6 497-500. 
9 Lorson CL, Strasswimmer J, Y ao J-M et al: SM N oligomerisation defect correlates with spinal muscular atrophy severity. Nat G enet 1998; 19: 63-66.

10 Liu Q, D reyfuss G: A novel nuclear structure containing the survival of motor neurons protein. E M BO J 1996; 15: 3555-3565.

11 Fischer U, L iu Q, D reyfuss G : The SM N-SIP1 complex has an essential role in spliceosomal snR NP biogenesis. Cell 1997; 90: 1023-1029.

12 Liu Q, Fischer U, D reyfuss G : The spinal muscular atrophy disease gene product (SMN) and its associated protein, SIP 1, are in a complex with spliceosomal snR N P proteins. Cell 1997; 90: 1013-1021.

13 Pellizzoni L, Kataoka N, Charroux B, D reyfuss G : A novel function for SM N, the Spinal M uscular A trophy disease gene product, in Pre-mRNA splicing. Cell 1998; 95: 615-624.
14 Coovert DD, Le TT, MCA ndrew PE et al: The survival motor neuron protein in spinal muscular atrophy. $\mathrm{Hum}$ M ol G enet 1997; 6: 1205-1214.

15 L efebvre S, Burlet P, Liu Q et al: Correlation between severity and SM N protein level in spinal muscular atrophy. $N$ at G enet 1997; 16: 265-269.

16 I wahashi $H$, E guchi $Y, Y$ asuhara N, H anafusa T, M atsuzawa $Y, T$ sujimoto $Y$ : Synergistic anti-apoptotic activity between $\mathrm{BCl}-2$ and SMN implicated in spinal muscular atrophy. Nature 1997; 390: 413-417.

17 Ponting C: Tudor domains in proteins that interact with R NA. Trend Biochem Sci 1997; 22: 51-52.

18 Talbot $K$, M iguel-A liaga I, Mohaghegh $P$, Ponting $C P$, $D$ avies K E : Characterisation of a gene encoding Survival $M$ otor N euron (SM N)-related protein, a constituent of the spliceosome complex. Hum Mol Genet 1998; 7 2149-2156. 